\title{
Chapter 8 \\ Foreign Investors vs. National Tax \\ Measures: Assessing the Role \\ of International Investment Agreements
}

\author{
Julien Chaisse and Jamieson Kirkwood
}

\subsection{Introduction}

The 2030 Agenda for Sustainable Development is an agreed blueprint for sustainable development, and its 17 Sustainable Development Goals (SDGs) are the means to facilitate this. The SDGs neither conflict with nor hinder taxation or investment policies. At the same time, the globalization of trade and investment has profoundly affected the practices and policies of international taxation. Indeed, tax professionals now require cross-disciplinary expertise to adequately understand the challenges faced by investors making investments across borders.

Moreover, in recent decades there has been a significant removal of many of the non-tax barriers to cross-border trade and investment. Firstly, international organizations, such as the World Trade Organization (WTO), the International Monetary Fund (IMF), the World Bank, and the Organization for Economic Cooperation and Development (OECD), have been pushing trade liberalization policies and the removal of exchange controls and controls on inward and outward investments (See Ring 2010). Additionally, the rapid proliferation of free trade agreements, which have removed or reduced the customs duties and tariffs on inward and outward transactions, reduced non-tax barriers and put in place trade facilitation mechanisms which has also had an enormous impact.

Indeed, most countries have entered into IIAs, and where the IIAs operate, investment-related activity such as Foreign Direct Investment (FDI) or NTMs will

J. Chaisse ( $\square)$

School of Law, City University of Hong Kong, Hong Kong, China

e-mail: julien.chaisse@cityu.edu.hk

J. Kirkwood

Faculty of Law, Chinese University of Hong Kong, Hong Kong, China

e-mail: Jamieson.Kirkwood@ link.cuhk.edu.hk 
become subject to the regulations provided in the IIA. This means that NTMs can therefore become breaches of IIAs, where the NTMs are deemed to conflict with the specific IIA. According to UNCTAD, there are currently 2659 active IIAs.

Additionally, the investment disputes which have dealt with tax issues will be identified, and it will also be demonstrated that both international investment law and investment arbitration, albeit unintentionally, significantly contribute to the regulatory framework applicable to tax policies. This chapter both identifies the theoretical convergence between tax and investment regimes and also identifies the actual provisions of IIAs that national policy-makers should observe and comply with in order to avoid investment arbitration (see Swenson et al. 2011).

\subsection{When Foreign Investment Meets Tax}

To determine whether an IIA is applicable to tax matters, the basic question is whether a given investment subject to NTMs constitutes a "foreign investment" within the meaning of that IIA. If yes, the investment will trigger the application of the IIA, which provides the framework for assessing the foreign treatment in the host state, including the treatment in terms of taxation (see Vieira 2014).

It is important to remember that IIAs are very different from Double Taxation Avoidance Agreements (DTAAs). While DTAAs allocate the taxing jurisdiction between the source and residence countries, in contrast, IIAs are not intended to provide specific taxation measures.

\subsubsection{The Broad Notion of Investment in IIAs}

The concept of "investment" does not have a generally accepted definition but will rather be specific to each treaty. Often, IIAs adopt a broad definition of "investment" that refers to "every kind of asset," "both tangible and intangible," of a foreign investor in a host country, suggesting that any economic activity is covered by that IIA. (Note also some IIAs have also focused on foreign investment in an "enterprise" rather than in a variety of assets.) The effect is the great scope of application of the related norms. Correspondingly, "foreign investment" is protected, with respect to all types of treatment adopted by the host state, and therefore tax is brought under the umbrella of IIAs.

\subsubsection{The Diversity of Tax Exceptions in IIAs}

As control over taxation matters is generally seen as a fundamental aspect of sovereignty, states' fiscal policies are generally excluded from IIAs' scope of 
Table 8.1 Typology of tax exceptions in IIAs

\begin{tabular}{|c|c|c|}
\hline Type of exclusion & Examples & Legal effect \\
\hline General exclusion & $\begin{array}{l}\text { "The provisions of this Agree- } \\
\text { ment shall not apply to matters of } \\
\text { taxation in the area of either } \\
\text { Contracting Party. Such matters } \\
\text { shall be governed by the domestic } \\
\text { laws of each Contracting Party } \\
\text { and the terms of any agreement } \\
\text { relating to taxation concluded } \\
\text { between the Contracting Parties." } \\
\text { Agreement between the Govern- } \\
\text { ment of Hong Kong and the } \\
\text { Government of New Zealand for } \\
\text { the Promotion and Protection of } \\
\text { Investments, H.K.-N.Z., art. } 8.2 \text {, } \\
\text { July } 6,1995,1889 \text { U.N.T.S. } 333\end{array}$ & $\begin{array}{l}\text { Such a provision excludes tax } \\
\text { matters from the treaty scope of } \\
\text { application without any reserva- } \\
\text { tion. It is impossible to bring a } \\
\text { tax-related dispute before an } \\
\text { investment tribunal on the } \\
\text { ground of such a treaty }\end{array}$ \\
\hline $\begin{array}{l}\text { General exclusion (but } \\
\text { with reservations for } \\
\text { EXP and compensation) }\end{array}$ & $\begin{array}{l}\text { "Nothing in this Agreement shall } \\
\text { apply to taxation measures except } \\
\text { as expressly provided for in para- } \\
\text { graphs } 3,4 \text { and 5." Article } 21 \text {, } \\
\text { China-Japan-Korea, Republic of } \\
\text { Trilateral Investment Agreement } \\
2012\end{array}$ & $\begin{array}{l}\text { Such a provision excludes tax } \\
\text { matters from the treaty scope of } \\
\text { application but with a reserva- } \\
\text { tion. The caveat is that disputes } \\
\text { relating to EXP and compensa- } \\
\text { tion can be brought to ISDS, and } \\
\text { therefore it is crucial to determine } \\
\text { if a tax measure is expropriatory } \\
\text { or not }\end{array}$ \\
\hline
\end{tabular}

Source: Compiled by the authors

application. However, this does not mean that investment arbitrators do not decide upon tax measures. IIAs might contain tax exceptions, but these are rather complex and often misunderstood.

Exclusions for tax measures are provided as follows:

1. Some IIAs differentiate between direct and indirect tax measures and often only subject the latter to the IIA regime.

2. Other IIAs rely on different exceptions, such as limiting the scope to national treatment (NT), most favored nation (MFN) treatment, fair and equitable treatment (FET), or a combination of all of these (see Stephan 2010).

Examples as regards the typology of tax exceptions are provided in the Table 8.1. Differing exceptions (or no exceptions) are provided by the specific treaty. Consequently, those IIAs that do not provide adequate tax exceptions may generate tax disputes under the treaty. 


\subsubsection{Interpretation by Investment Tribunals}

The complexity has been added to by investment tribunals that have also developed specific, although inconsistent, approaches to the interpretation of taxation exclusions. For instance, the Occidental Exploration v. Ecuador Final Award interprets a taxation provision to permit claims based on the bilateral investment treaty's (BIT) FET standard. However, the Pan American Energy LLC and BP Argentina Exploration Company v. The Argentine Republic case disagrees with the approach taken in Occidental Exploration v. Ecuador. Significantly, the Burlington Resources Inc. v. Republic of Ecuador case holds that under Article X of the BIT, "matters of taxation" are as a rule excluded from the scope of the treaty and examines the claims advanced by the claimant individually in order to ascertain whether the taxation law is challenged or not with respect to each claim. Also, the Nations Energy Corporation v. Panama case interprets the BIT to exclude claims stemming from taxation matters based on the FET standard. Finally, in a very straightforward manner, the Quasar de Valors v. Russia Award on Preliminary Objections notes that a taxation exclusion cannot provide a loophole to escape the central undertakings of investor protection.

\subsubsection{The Multitude of Tax Disputes Before Investment Tribunals}

Nevertheless, despite this enormous complexity, there is a multitude of tax disputes before international investment tribunals, which represents a significant change in the landscape of international investment. This can partly be explained because, as described below, the standard dispute resolution mechanism of international tax treaties is generally unsatisfactory to the investor.

\subsubsection{Tax Treaty Dispute Resolution Mechanisms}

Either:

1. The Mutual Agreement Procedures (MAPs) but investors have to go through a lengthy process and are not guaranteed to have a case heard by an arbitration tribunal (Arts. 25 (1) and (2) of the OECD Model Convention provide the regulations on how investors should proceed during tax disputes). Also other drawbacks include a lack of transparency (i.e., how the tax authority makes the decision to accept or reject the case is not disclosed); procedural inefficiencies (e.g., the MAPs impose a relaxed responsibility on the competent authority, who just needs to "endeavor" to settle the controversy but is not "obliged" to settle the 
dispute); no right for taxpayers/investors to be treated fairly; and an inherent inability to resolve issues of double taxation, transfer pricing, etc. (This is because the MAPs fail to determine the allocation of taxes each contracting country should receive.)

2. The OECD Model Tax Convention, if applicable, does provide the possibility of arbitration (see Christians 2009). However, similarly, an investor also cannot directly access arbitration and must go through a difficult process (the investor must first qualify under Arts. 25(1) and (2) of the OECD Model Tax Convention). Although this might expand, the arbitration clause serves only as an extension of the MAPs (and only for the issues which cannot be solved in the MAPs process but not for the whole dispute as stated in Art. 25(5) of OECD Model Tax Convention). The arbitration clause is a supplement to the MAPs and cannot replace them. The OECD has commented that the arbitration clause is an "additional dispute resolution technique which can help to ensure that international tax disputes will, to the greatest extent possible, be resolved in a final, principled, fair and objective manner for both the countries and the taxpayers concerned." In fact this OECD sees this as a bar on applying for arbitration if the local court has already resolved the tax dispute. For this reason, the Art. 25(5) of the OECD Model Tax Convention avoids bestowing parallel authority (i.e., to a domestic court and an international tribunal) to deal with the matter. Another interpretation of the arbitration clause is that the investor has to waive the right to access the domestic courts in order to request that tax disputes be submitted to arbitration under the OECD Model Convention or international tax treaties (to avoid parallel authority), a 2-year waiting period (the waiting period stated in Art. 25(2) of the OECD Model Tax Convention), representation only at the state level (The investor is excluded from being a claimant in arbitration and standing is given to the contacting state's competent authority, i.e. the tax authority) and an inadequate enforcement regime. In this case, the decision only binds the two contracting states, and if the losing contracting state does not comply with the arbitral decision, the winning contracting state or the taxpayer/investor can do nothing. Remarkably, there is no enforcement mechanism under the international tax treaties, and no sanction or confiscating measures can be imposed upon the losing contracting state for any non-compliance of the arbitral decision.

These unsatisfactory processes are an exogenous factor explaining the multitude of tax disputes before investment tribunals.

\subsubsection{Other Endogenous Factors}

The two main additional factors explaining the multitude of tax disputes before investment tribunals are as follows: 
1. One of the key features of investment protection consists of allowing foreign investors to challenge the host government's actions before an international arbitral court.

2. The expansion of foreign investment into an ever-increasing number of types of investments inevitably generates tension with host states that may result in innovative and complicated disputes that an arbitral tribunal is asked to resolve.

\subsection{The Judicial Review of Tax Regulations by Investment Tribunals}

Unsurprisingly, therefore, since 1999, at least 32 tax-related cases have been brought to international arbitration. However, this number is likely to be only the tip of the iceberg as many arbitration cases remain unknown to the public, are not disclosed, or are still being negotiated.

\subsubsection{Tax Disputes Not Won by the Investor}

Not all investment claims result in an award that the host state will have to pay. In fact, the data shows that slightly more than $50 \%$ of the investment claims dealing with tax matters resulted in a tribunal decision in favor of the state. This article has identified 17 disputes that resulted in a decision denying a breach of the relevant IIA. This first category of decisions indicates that foreign investors considered using IIAs to submit claims against a number of countries and tax measures. Such a trend shows that IIAs are a potential recourse against some domestic tax measures. Table 8.2 provides the details of each of these 17 disputes.

In these 17 disputes, there is a great diversity of tax measures that were at the origin of the dispute, namely, windfall profits tax, tax investigations, value-added tax, taxation of income trusts, import taxes, corporate income tax, tax stamps on cigarettes, duty-free regime, etc. Unsurprisingly, the broad scope of application of IIAs allows tribunals to look at a wide variety of tax measures.

There is also diversity in the countries involved in these disputes since the countries do not belong to the same economic category. That being said, it is hard to expand the analysis further because, in most of these disputes, the states have proven that they use tax laws or regulations in a manner compatible with the relevant IIA. Alternatively, if the tribunal rejected the claim for lack of jurisdiction, nothing can be concluded as to the potential breach of investment law by a domestic tax measure. The scenario is radically different when one looks at the disputes lost by various host states. 
Table 8.2 Investment disputes not won by the investor

\begin{tabular}{|c|c|c|c|c|c|}
\hline Case name & Tax area & Treaty & $\begin{array}{l}\text { Year } \\
\text { of } \\
\text { claim }\end{array}$ & $\begin{array}{l}\text { Award } \\
\text { date }\end{array}$ & Outcome \\
\hline $\begin{array}{l}\text { Corn Products } \\
\text { International } \\
\text { Inc. v. Mexican } \\
\text { States }\end{array}$ & $\begin{array}{l}\text { Imposition of a } \\
\text { new tax on soft } \\
\text { drinks and syrups } \\
\text { sweetened by arti- } \\
\text { ficial sweeteners }\end{array}$ & NAFTA & 2003 & $\begin{array}{l}\text { August } \\
18,2009\end{array}$ & Not public \\
\hline $\begin{array}{l}\text { Paushok } \\
\text { v. Mongolia }\end{array}$ & $\begin{array}{l}\text { Resource man- } \\
\text { agement (oil and } \\
\text { gas), tax (windfall } \\
\text { profits tax), } \\
\text { employment (per- } \\
\text { formance } \\
\text { requirements) }\end{array}$ & $\begin{array}{l}\text { Russia-Mon- } \\
\text { golia BIT }\end{array}$ & 2007 & $\begin{array}{l}\text { April } \\
28,2011\end{array}$ & $\begin{array}{l}\text { Tribunal } \\
\text { accepted jurisdic- } \\
\text { tion over claims, } \\
\text { denied claims } \\
\text { except the taking } \\
\text { of the gold. The } \\
\text { claimants had } \\
60 \text { days to claim } \\
\text { damages }\end{array}$ \\
\hline $\begin{array}{l}\text { Burlington } \\
\text { Resources } \\
\text { v. Ecuador }\end{array}$ & $\begin{array}{l}\text { Windfall profits } \\
\text { tax, enforcement } \\
\text { of that tax, physi- } \\
\text { cal takeover of the } \\
\text { oil fields }\end{array}$ & $\begin{array}{l}\text { USA- } \\
\text { ECUADOR }\end{array}$ & 2008 & $\begin{array}{l}\text { December } \\
14,2012\end{array}$ & $\begin{array}{l}\text { Tribunal rejected } \\
\text { jurisdiction over } \\
\text { umbrella clause } \\
\text { but accepted } \\
\text { jurisdiction over } \\
\text { caucidad } \\
\text { decrees. Tribunal } \\
\text { decided Ecuador } \\
\text { expropriated } \\
\text { claimant's } \\
\text { investment } \\
\text { unlawfully. Other } \\
\text { claims are } \\
\text { dismissed }\end{array}$ \\
\hline $\begin{array}{l}\text { Phoenix Action } \\
\text { v. Czech } \\
\text { Republic }\end{array}$ & $\begin{array}{l}\text { Administration of } \\
\text { justice (court } \\
\text { decisions), tax } \\
\text { (investigations), } \\
\text { border control } \\
\text { (customs) }\end{array}$ & $\begin{array}{l}\text { Croatia- } \\
\text { Czech Rep } \\
\text { BIT }\end{array}$ & 2004 & $\begin{array}{l}\text { April } \\
15,2009\end{array}$ & $\begin{array}{l}\text { Tribunal rejected } \\
\text { jurisdiction over } \\
\text { claim. The } \\
\text { claimant had to } \\
\text { pay all arbitration } \\
\text { costs }\end{array}$ \\
\hline $\begin{array}{l}\text { Noble Energy } \\
\text { v. Ecuador }\end{array}$ & $\begin{array}{l}\text { Utilities (electric- } \\
\text { ity), privatization } \\
\text { (energy), tax } \\
\text { (value-added tax), } \\
\text { public order } \\
\text { (enforcement of } \\
\text { electricity rates), } \\
\text { energy (subsidies) }\end{array}$ & $\begin{array}{l}\text { USA Ecua- } \\
\text { dor BIT }\end{array}$ & 2005 & Settled & $\begin{array}{l}\text { Tribunal } \\
\text { accepted jurisdic- } \\
\text { tion. Case subse- } \\
\text { quently settled by } \\
\text { agreement } \\
\text { between claimant } \\
\text { and respondent } \\
\text { state }\end{array}$ \\
\hline $\begin{array}{l}\text { Gottlieb v. } \\
\text { Canada }\end{array}$ & $\begin{array}{l}\text { Tax (taxation of } \\
\text { income trusts), } \\
\text { resource manage- } \\
\text { ment (oil and gas) }\end{array}$ & NAFTA & 2007 & & $\begin{array}{l}\text { Claim rejected } \\
\text { following agree- } \\
\text { ment of respon- } \\
\text { dent state and } \\
\text { claimant's state }\end{array}$ \\
\hline
\end{tabular}


Table 8.2 (continued)

\begin{tabular}{|c|c|c|c|c|c|}
\hline Case name & Tax area & Treaty & $\begin{array}{l}\text { Year } \\
\text { of } \\
\text { claim }\end{array}$ & $\begin{array}{l}\text { Award } \\
\text { date }\end{array}$ & Outcome \\
\hline & & & & & $\begin{array}{l}\text { of nationality that } \\
\text { taxation measure } \\
\text { did not constitute } \\
\text { an expropriation }\end{array}$ \\
\hline $\begin{array}{l}\text { TCW v Domini- } \\
\text { can Republic }\end{array}$ & $\begin{array}{l}\text { Utilities (electric- } \\
\text { ity), privatization } \\
\text { (energy), public } \\
\text { order (theft of } \\
\text { electricity), tax } \\
\text { (investigations) }\end{array}$ & CAFTA & 2007 & $\begin{array}{l}\text { Consent } \\
\text { Award/ } \\
\text { July } \\
16,2009\end{array}$ & $\begin{array}{l}\text { Case settled by } \\
\text { agreement of } \\
\text { claimant and } \\
\text { respondent state } \\
\text { before any deci- } \\
\text { sion on } \\
\text { jurisdiction }\end{array}$ \\
\hline $\begin{array}{l}\text { Lacich v } \\
\text { Canada }\end{array}$ & $\begin{array}{l}\text { Tax (taxation of } \\
\text { income trusts), } \\
\text { resource manage- } \\
\text { ment (oil and gas) }\end{array}$ & NAFTA & 2009 & Withdrawn & $\begin{array}{l}\text { Claim withdrawn } \\
\text { before tribunal } \\
\text { established }\end{array}$ \\
\hline $\begin{array}{l}\text { Link-Trading } v \\
\text { Moldova }\end{array}$ & $\begin{array}{l}\text { Tax (import } \\
\text { taxes), border } \\
\text { control (customs), } \\
\text { industrial policy } \\
\text { (free economic } \\
\text { zones) }\end{array}$ & $\begin{array}{l}\text { USA Mol- } \\
\text { dova BIT }\end{array}$ & 1999 & $\begin{array}{l}\text { April } \\
18,2002\end{array}$ & $\begin{array}{l}\text { Tribunal } \\
\text { accepted jurisdic- } \\
\text { tion over claim } \\
\text { but decided that } \\
\text { respondent state } \\
\text { did not violate } \\
\text { treaty. The } \\
\text { claimant was } \\
\text { required to pay } \\
\text { toward legal } \\
\text { costs of } \\
\text { respondent }\end{array}$ \\
\hline $\begin{array}{l}\text { Tokios Tokeles } \\
\text { v. Ukraine }\end{array}$ & $\begin{array}{l}\text { Culture (print } \\
\text { publishing), tax } \\
\text { (investigations) }\end{array}$ & $\begin{array}{l}\text { Lithuania- } \\
\text { Ukraine BIT }\end{array}$ & 2002 & $\begin{array}{l}\text { July } \\
26,2007\end{array}$ & $\begin{array}{l}\text { Tribunal } \\
\text { accepted jurisdic- } \\
\text { tion over claim } \\
\text { but decided that } \\
\text { respondent state } \\
\text { did not violate } \\
\text { treaty. Tribunal } \\
\text { split arbitration } \\
\text { costs between } \\
\text { claimant and } \\
\text { respondent state }\end{array}$ \\
\hline $\begin{array}{l}\text { EnCana } \\
\text { v. Ecuador }\end{array}$ & $\begin{array}{l}\text { Tax (value-added } \\
\text { tax), public } \\
\text { contracting (oil } \\
\text { production), } \\
\text { resource manage- } \\
\text { ment (oil) }\end{array}$ & $\begin{array}{l}\text { Canada- } \\
\text { Ecuador BIT }\end{array}$ & 2003 & $\begin{array}{l}\text { February } \\
3,2006\end{array}$ & $\begin{array}{l}\text { Tribunal rejected } \\
\text { jurisdiction over } \\
\text { claim except } \\
\text { expropriation, } \\
\text { decided that } \\
\text { respondent state } \\
\text { did not violate } \\
\text { treaty. The } \\
\text { respondent state }\end{array}$ \\
\hline
\end{tabular}


Table 8.2 (continued)

\begin{tabular}{|c|c|c|c|c|c|}
\hline Case name & Tax area & Treaty & $\begin{array}{l}\text { Year } \\
\text { of } \\
\text { claim }\end{array}$ & $\begin{array}{l}\text { Award } \\
\text { date }\end{array}$ & Outcome \\
\hline & & & & & $\begin{array}{l}\text { had to pay all } \\
\text { arbitration costs }\end{array}$ \\
\hline $\begin{array}{l}\text { Plama Consor- } \\
\text { tium Limited } v . \\
\text { Bulgaria }\end{array}$ & $\begin{array}{l}\text { Environmental } \\
\text { protection (envi- } \\
\text { ronmental liabil- } \\
\text { ity), administra- } \\
\text { tion of justice } \\
\text { (bankruptcy } \\
\text { administration), } \\
\text { public order } \\
\text { (occupations of oil } \\
\text { refinery), tax (cor- } \\
\text { porate income } \\
\text { tax), privatization } \\
\text { (oil refinery) }\end{array}$ & $\begin{array}{l}\text { ECT and } \\
\text { Bulgaria- } \\
\text { Cyprus BIT }\end{array}$ & 2003 & $\begin{array}{l}\text { August } \\
27,2008\end{array}$ & $\begin{array}{l}\text { Tribunal } \\
\text { accepted jurisdic- } \\
\text { tion over claims } \\
\text { arising from ECT } \\
\text { but decided that } \\
\text { the claimant is } \\
\text { not entitled to } \\
\text { any of the sub- } \\
\text { stantive protec- } \\
\text { tions provided by } \\
\text { the ECT and } \\
\text { respondent state } \\
\text { did not violate } \\
\text { treaty. Tribunal } \\
\text { required claimant } \\
\text { to pay all arbitra- } \\
\text { tion costs as well } \\
\text { as legal costs of } \\
\text { respondent state }\end{array}$ \\
\hline $\begin{array}{l}\text { Grand River } \\
\text { v. USA }\end{array}$ & $\begin{array}{l}\text { Public health } \\
\text { (anti-smoking), } \\
\text { administration of } \\
\text { justice (settlement } \\
\text { with cigarette } \\
\text { manufacturers), } \\
\text { tax (tax stamps on } \\
\text { cigarettes) }\end{array}$ & NAFTA & 2004 & $\begin{array}{l}\text { January } \\
12,2011\end{array}$ & $\begin{array}{l}\text { Tribunal rejected } \\
\text { jurisdiction over } \\
\text { claims of Grand } \\
\text { River, accepted } \\
\text { jurisdiction over } \\
\text { claims of Arthur } \\
\text { Montour but } \\
\text { decided that the } \\
\text { respondent state } \\
\text { did not violate } \\
\text { the treaty and } \\
\text { split the arbitra- } \\
\text { tion costs }\end{array}$ \\
\hline $\begin{array}{l}\text { Amto } \\
\text { LLC. v. Ukraine }\end{array}$ & $\begin{array}{l}\text { Tax procedure } \\
\text { (energy, industrial } \\
\text { policy, } \\
\text { privatization) }\end{array}$ & ECT & 2005 & $\begin{array}{l}\text { March } \\
26,2008\end{array}$ & $\begin{array}{l}\text { Tribunal } \\
\text { accepted jurisdic- } \\
\text { tion over claim, } \\
\text { but decided that } \\
\text { respondent state } \\
\text { did not violate } \\
\text { treaty. The arbi- } \\
\text { tration costs were } \\
\text { split between the } \\
\text { parties and each } \\
\text { party bore its } \\
\text { own legal costs }\end{array}$ \\
\hline
\end{tabular}


Table 8.2 (continued)

\begin{tabular}{|c|c|c|c|c|c|}
\hline Case name & Tax area & Treaty & $\begin{array}{l}\text { Year } \\
\text { of } \\
\text { claim }\end{array}$ & $\begin{array}{l}\text { Award } \\
\text { date }\end{array}$ & Outcome \\
\hline EDF v. Romania & $\begin{array}{l}\text { Public order (cor- } \\
\text { ruption investiga- } \\
\text { tions), border } \\
\text { control (customs), } \\
\text { tax (duty-free } \\
\text { regime), interna- } \\
\text { tional relations } \\
\text { (European Union } \\
\text { accession) }\end{array}$ & $\begin{array}{l}\text { UK-Romania } \\
\text { BIT }\end{array}$ & 2005 & $\begin{array}{l}\text { October } \\
8,2009\end{array}$ & $\begin{array}{l}\text { Tribunal } \\
\text { accepted jurisdic- } \\
\text { tion over claim } \\
\text { but decided that } \\
\text { respondent state } \\
\text { did not violate } \\
\text { treaty. Tribunal } \\
\text { split arbitration } \\
\text { costs between } \\
\text { claimant and } \\
\text { respondent state } \\
\text { but required } \\
\text { claimant to pay } \\
\$ 6 \text { million toward } \\
\text { legal costs of } \\
\text { respondent state }\end{array}$ \\
\hline $\begin{array}{l}\text { The Rompetrol } \\
\text { Group } \\
\text { N.V. v. Romania }\end{array}$ & $\begin{array}{l}\text { Irregularities dur- } \\
\text { ing the privatiza- } \\
\text { tion, tax fraud, } \\
\text { corruption, abuse } \\
\text { of power, money } \\
\text { laundering }\end{array}$ & $\begin{array}{l}\text { Netherlands- } \\
\text { Romania BIT }\end{array}$ & 2005 & $\begin{array}{l}\text { May } \\
6,2013\end{array}$ & $\begin{array}{l}\text { The tribunal } \\
\text { accepted jurisdic- } \\
\text { tion over claims, } \\
\text { decided that there } \\
\text { was no breach of } \\
\text { the treaty }\end{array}$ \\
\hline $\begin{array}{l}\text { Jan Oostergetel } \\
\text { and Theodora } \\
\text { Laurentius } \\
\text { v. The Slovak } \\
\text { Republic }\end{array}$ & $\begin{array}{l}\text { Bankruptcy, tax } \\
\text { arrears }\end{array}$ & $\begin{array}{l}\text { Netherlands- } \\
\text { Slovak } \\
\text { Republic BIT }\end{array}$ & 2006 & $\begin{array}{l}\text { April } \\
23,2012\end{array}$ & $\begin{array}{l}\text { Tribunal decided } \\
\text { that respondent } \\
\text { state did not } \\
\text { breach treaty, } \\
\text { ordered the } \\
\text { claimants to bear } \\
\text { the arbitration } \\
\text { costs and to pay } \\
\text { the respondent } \\
2 \text { million euros as } \\
\text { contribution to } \\
\text { legal and other } \\
\text { costs }\end{array}$ \\
\hline
\end{tabular}

Source: International Investment Arbitration and Public Policy (IIAPP) Database (available at http://www.iiapp.org ) and relevant awards. Table compiled by authors

\subsubsection{Tax Disputes Lost by the Host States}

Out of the 32 disputes dealing with tax matters, 15 have been lost by the host states. These disputes are the most interesting because they show what can go wrong in terms of designing tax policy in accordance with IIAs. Table 8.3 produces the details of each of these 15 disputes. 
Table 8.3 Investment disputes lost by host states

\begin{tabular}{|c|c|c|c|c|c|}
\hline Case name & Tax area & Treaty & $\begin{array}{l}\text { Year } \\
\text { of } \\
\text { claim }\end{array}$ & $\begin{array}{l}\text { Award } \\
\text { date }\end{array}$ & Outcome \\
\hline $\begin{array}{l}\text { Feldman } \\
\text { v. Mexico }\end{array}$ & $\begin{array}{l}\text { Tax (excise tax on } \\
\text { cigarettes), border } \\
\text { control (customs, } \\
\text { gray market } \\
\text { exports of } \\
\text { cigarettes) }\end{array}$ & NAFTA & 1999 & $\begin{array}{l}\text { December } \\
16,2002\end{array}$ & $\begin{array}{l}\text { Tribunal } \\
\text { accepted juris- } \\
\text { diction over } \\
\text { claim, decided } \\
\text { that respondent } \\
\text { state violated } \\
\text { treaty }(\mathrm{NT}) \text {. The } \\
\text { court awarded } \\
\text { specifically } \\
\text { “\$16,961,056 } \\
\text { Mexican pesos } \\
\text { (principal } \\
\text { amount of } \\
\$ 9,464,627.50 \\
\text { plus interest of } \\
\$ 7,496,428.47) \text {. } \\
\text { Tribunal split } \\
\text { arbitration costs } \\
\text { between claimant } \\
\text { and respondent } \\
\text { state }\end{array}$ \\
\hline Goetz v. Burundi & $\begin{array}{l}\text { Cancellation of } \\
\text { license to operate } \\
\text { in a free eco- } \\
\text { nomic zone }\end{array}$ & $\begin{array}{l}\text { Belgium- } \\
\text { Luxemburg- } \\
\text { Burundi BIT }\end{array}$ & 1999 & $\begin{array}{l}\text { February } \\
10,1999\end{array}$ & $\begin{array}{l}\text { Indirect expro- } \\
\text { priation subject } \\
\text { to compensation } \\
\text { amounted to } \\
\text { roughly } \$ 3 \\
\text { million }\end{array}$ \\
\hline $\begin{array}{l}\text { Enron Corpora- } \\
\text { tion \& Ponderosa } \\
\text { Assets LP v. The } \\
\text { Argentine } \\
\text { Republic }\end{array}$ & Stamp tax & $\begin{array}{l}\text { Argentine- } \\
\text { USA BIT }\end{array}$ & 2001 & $\begin{array}{l}\text { May } \\
22,2007\end{array}$ & $\begin{array}{l}\text { Tribunal decided } \\
\text { that Argentina } \\
\text { breached FET } \\
\text { and umbrella } \\
\text { clauses and } \\
\text { awarded a com- } \\
\text { pensation of } \\
\$ 106.2 \text { million }\end{array}$ \\
\hline $\begin{array}{l}\text { Occidental } \\
\text { Exploration and } \\
\text { Production Com- } \\
\text { pany v. Ecuador } \\
\text { Case No. UN } \\
3467\end{array}$ & $\begin{array}{l}\text { Tax (value-added } \\
\text { tax), public } \\
\text { contracting (oil } \\
\text { production) }\end{array}$ & $\begin{array}{l}\text { USA-Ecua- } \\
\text { dor BIT }\end{array}$ & 2002 & $\begin{array}{l}\text { July } \\
1,2004\end{array}$ & $\begin{array}{l}\text { Tribunal } \\
\text { accepted juris- } \\
\text { diction over } \\
\text { claim, decided } \\
\text { that respondent } \\
\text { state violated } \\
\text { treaty (NT and } \\
\text { FET), and } \\
\text { awarded approxi- } \\
\text { mately \$75.0 } \\
\text { million (plus } \\
\text { interest) against } \\
\text { respondent state. }\end{array}$ \\
\hline
\end{tabular}


Table 8.3 (continued)

\begin{tabular}{|c|c|c|c|c|c|}
\hline Case name & Tax area & Treaty & $\begin{array}{l}\text { Year } \\
\text { of } \\
\text { claim }\end{array}$ & $\begin{array}{l}\text { Award } \\
\text { date }\end{array}$ & Outcome \\
\hline & & & & & $\begin{array}{l}\text { Tribunal required } \\
\text { respondent state } \\
\text { to pay } 55 \% \text { of } \\
\text { arbitration costs }\end{array}$ \\
\hline $\begin{array}{l}\text { Archer Daniels } \\
\text { Midland Co. \& } \\
\text { Tate Lyle Ingre- } \\
\text { dients Americas, } \\
\text { Inc. v. United } \\
\text { Mexican States }\end{array}$ & $\begin{array}{l}\text { Imposition of a } \\
\text { new tax on soft } \\
\text { drinks and syrups } \\
\text { sweetened by } \\
\text { sweeteners other } \\
\text { than sugar }\end{array}$ & NAFTA & 2003 & $\begin{array}{l}\text { November } \\
21,2007\end{array}$ & $\begin{array}{l}\text { Tribunal decided } \\
\text { that Mexico } \\
\text { breached articles } \\
\text { on NT and per- } \\
\text { formance } \\
\text { requirement and } \\
\text { that the tax } \\
\text { imposed does not } \\
\text { amount to a valid } \\
\text { countermeasure. } \\
\text { Tribunal awarded } \\
\text { US } \$ 33.0 \text { million }\end{array}$ \\
\hline $\begin{array}{l}\text { El Paso Energy } \\
\text { International } \\
\text { Company } \\
\text { v. Argentine } \\
\text { Republic }\end{array}$ & $\begin{array}{l}\text { Resource man- } \\
\text { agement (oil and } \\
\text { gas), utilities } \\
\text { (electricity), pri- } \\
\text { vatization } \\
\text { (energy), mone- } \\
\text { tary system } \\
\text { (financial crisis, } \\
\text { currency reform) }\end{array}$ & $\begin{array}{l}\text { Argentina- } \\
\text { USA BIT }\end{array}$ & 2003 & $\begin{array}{l}\text { October } \\
31,2011\end{array}$ & $\begin{array}{l}\text { Tribunal decided } \\
\text { that the Argen- } \\
\text { tine Republic } \\
\text { breached FET, } \\
\text { awarded a com- } \\
\text { pensation of } \\
\$ 43.0 \text { million } \\
\text { plus interest, split } \\
\text { the arbitration } \\
\text { costs. Parties } \\
\text { bear their own } \\
\text { legal costs and } \\
\text { expenses }\end{array}$ \\
\hline $\begin{array}{l}\text { Duke Energy } \\
\text { v. Ecuador }\end{array}$ & $\begin{array}{l}\text { Utilities (electric- } \\
\text { ity), public } \\
\text { contracting (elec- } \\
\text { tric power pro- } \\
\text { curement), border } \\
\text { control (customs), } \\
\text { tax (import taxes) }\end{array}$ & $\begin{array}{l}\text { USA-Ecua- } \\
\text { dor BIT }\end{array}$ & 2004 & $\begin{array}{l}\text { August } \\
18,2008\end{array}$ & $\begin{array}{l}\text { Tribunal } \\
\text { accepted juris- } \\
\text { diction over } \\
\text { claim except cus- } \\
\text { tom duties, } \\
\text { decided that } \\
\text { respondent state } \\
\text { violated treaty } \\
\text { (NT and } \\
\text { umbrella clause), } \\
\text { and awarded } \\
\text { approximately } \\
\$ 5.6 \text { million (plus } \\
\text { interest) against } \\
\text { respondent state. } \\
\text { Tribunal split } \\
\text { arbitration costs } \\
\text { between claimant }\end{array}$ \\
\hline
\end{tabular}


Table 8.3 (continued)

\begin{tabular}{|c|c|c|c|c|c|}
\hline Case name & Tax area & Treaty & $\begin{array}{l}\text { Year } \\
\text { of } \\
\text { claim }\end{array}$ & $\begin{array}{l}\text { Award } \\
\text { date }\end{array}$ & Outcome \\
\hline & & & & & $\begin{array}{l}\text { and respondent } \\
\text { state }\end{array}$ \\
\hline Hulley v. Russia & $\begin{array}{l}\text { Tax (tax evasion } \\
\text { investigations), } \\
\text { public order (sei- } \\
\text { zures of facili- } \\
\text { ties), administra- } \\
\text { tion of justice } \\
\text { (court decisions), } \\
\text { privatization (oil } \\
\text { industry) }\end{array}$ & ECT & 2005 & $\begin{array}{l}\text { July } \\
18,2014\end{array}$ & $\begin{array}{l}\text { Tribunal } \\
\text { accepted juris- } \\
\text { diction, decided } \\
\text { that Russia } \\
\text { breached expro- } \\
\text { priation article, } \\
\text { awarded dam- } \\
\text { ages of } \$ 40 \\
\text { billion }\end{array}$ \\
\hline $\begin{array}{l}\text { RosInvestCo v. } \\
\text { Russia }\end{array}$ & $\begin{array}{l}\text { Tax (tax evasion } \\
\text { investigations), } \\
\text { public order (sei- } \\
\text { zures of facili- } \\
\text { ties), administra- } \\
\text { tion of justice } \\
\text { (court decisions), } \\
\text { privatization (oil } \\
\text { industry) }\end{array}$ & $\begin{array}{l}\text { UK-USSR } \\
\text { BIT }\end{array}$ & 2005 & $\begin{array}{l}\text { December } \\
22,2010\end{array}$ & $\begin{array}{l}\text { Tribunal } \\
\text { accepted juris- } \\
\text { diction, decided } \\
\text { that Russia } \\
\text { breached expro- } \\
\text { priation article, } \\
\text { awarded dam- } \\
\text { ages of } \$ 3.5 \text { mil- } \\
\text { lion, split the } \\
\text { arbitration costs }\end{array}$ \\
\hline $\begin{array}{l}\text { Yukos Universal } \\
\text { v. Russia }\end{array}$ & $\begin{array}{l}\text { Tax (tax evasion } \\
\text { investigations), } \\
\text { public order (sei- } \\
\text { zures of facili- } \\
\text { ties), administra- } \\
\text { tion of justice } \\
\text { (court decisions), } \\
\text { privatization (oil } \\
\text { industry) }\end{array}$ & ECT & 2005 & $\begin{array}{l}\text { July } \\
18,2014\end{array}$ & $\begin{array}{l}\text { Tribunal } \\
\text { accepted juris- } \\
\text { diction, decided } \\
\text { that Russia } \\
\text { breached expro- } \\
\text { priation article, } \\
\text { awarded dam- } \\
\text { ages of } \$ 1.8 \\
\text { billion }\end{array}$ \\
\hline $\begin{array}{l}\text { Mobil } \\
\text { v. Venezuela }\end{array}$ & $\begin{array}{l}\text { Resource man- } \\
\text { agement (oil), } \\
\text { public contracting } \\
\text { (oil production), } \\
\text { tax (corporate } \\
\text { income tax) }\end{array}$ & $\begin{array}{l}\text { Netherlands- } \\
\text { Venezuela } \\
\text { BIT }\end{array}$ & 2007 & $\begin{array}{l}\text { October } \\
9,2014\end{array}$ & $\begin{array}{l}\text { The tribunal } \\
\text { accepted juris- } \\
\text { diction over } \\
\text { claims except the } \\
\text { claim arising out } \\
\text { of increase in the } \\
\text { income tax rate. } \\
\text { It awarded com- } \\
\text { pensation of } \\
\text { about } \$ 1411 \text { mil- } \\
\text { lion plus interest } \\
\text { for expropriation } \\
\text { and } \$ 9.0 \text { million } \\
\text { plus interest for } \\
\text { the production } \\
\text { and export cur- } \\
\text { tailments. It split }\end{array}$ \\
\hline
\end{tabular}


Table 8.3 (continued)

\begin{tabular}{|c|c|c|c|c|c|}
\hline Case name & Tax area & Treaty & $\begin{array}{l}\text { Year } \\
\text { of } \\
\text { claim }\end{array}$ & $\begin{array}{l}\text { Award } \\
\text { date }\end{array}$ & Outcome \\
\hline & & & & & $\begin{array}{l}\text { the arbitration } \\
\text { costs }\end{array}$ \\
\hline $\begin{array}{l}\text { Quasar de } \\
\text { Valores Sicav SA } \\
\text { v. The Russian } \\
\text { Federation }\end{array}$ & $\begin{array}{l}\text { Tax (tax evasion } \\
\text { investigations), } \\
\text { public order (sei- } \\
\text { zures of facili- } \\
\text { ties), administra- } \\
\text { tion of justice } \\
\text { (court decisions) }\end{array}$ & $\begin{array}{l}\text { Spain-USSR } \\
\text { BIT }\end{array}$ & 2007 & $\begin{array}{l}\text { July } \\
20,2012\end{array}$ & $\begin{array}{l}\text { Tribunal } \\
\text { accepted juris- } \\
\text { diction, decided } \\
\text { that Russia } \\
\text { breached expro- } \\
\text { priation article, } \\
\text { awarded dam- } \\
\text { ages of around } \\
\$ 2.0 \text { million in } \\
\text { total }\end{array}$ \\
\hline Renta 4 v. Russia & $\begin{array}{l}\text { Tax assessment, } \\
\text { public order (sei- } \\
\text { zures of facili- } \\
\text { ties), administra- } \\
\text { tion of justice } \\
\text { (court decisions), } \\
\text { privatization (oil } \\
\text { industry) }\end{array}$ & $\begin{array}{l}\text { Spain-USSR } \\
\text { BIT }\end{array}$ & 2007 & $\begin{array}{l}\text { March } \\
30,2009\end{array}$ & $\begin{array}{l}\text { Quasar case was } \\
\text { formerly known } \\
\text { as Renta } 4 \text { case. } \\
\text { In the jurisdic- } \\
\text { tional award, the } \\
\text { tribunal accepted } \\
\text { jurisdiction over } \\
\text { claims of only } \\
\text { four of the seven } \\
\text { claimants of the } \\
\text { Renta } 4 \text { case. } \\
\text { Therefore, the } \\
\text { case is renamed } \\
\text { as Quasar }\end{array}$ \\
\hline $\begin{array}{l}\text { Tza Yap Shum } \\
\text { v. Republic of } \\
\text { Peru }\end{array}$ & Tax assessment & $\begin{array}{l}\text { China-Peru } \\
\text { BIT }\end{array}$ & 2005 & $\begin{array}{l}\text { July } \\
7,2011\end{array}$ & $\begin{array}{l}\text { Expropriation } \\
\text { case is not avail- } \\
\text { able in English }\end{array}$ \\
\hline $\begin{array}{l}\text { Veteran Petro- } \\
\text { leum v. Russia }\end{array}$ & $\begin{array}{l}\text { Tax (tax evasion } \\
\text { investigations), } \\
\text { public order (sei- } \\
\text { zures of facili- } \\
\text { ties), administra- } \\
\text { tion of justice } \\
\text { (court decisions), } \\
\text { privatization (oil } \\
\text { industry) }\end{array}$ & ECT & 2005 & $\begin{array}{l}\text { July } \\
18,2014\end{array}$ & $\begin{array}{l}\text { Tribunal } \\
\text { accepted juris- } \\
\text { diction, decided } \\
\text { that Russia } \\
\text { breached expro- } \\
\text { priation article, } \\
\text { awarded dam- } \\
\text { ages of } \\
\$ 50,020,867,798\end{array}$ \\
\hline
\end{tabular}

Source: International Investment Arbitration and Public Policy (IIAPP) Database (available at http://www.iiapp.org ) and relevant awards. Table compiled by authors

These 15 disputes have been lost by only 7 countries, all of which are developing countries or transition economies.

The majority of cases (nine awards) concluded with a finding of expropriation. However, two claims were consolidated into a single case for Renta 4 and Quasar de Valores v. Russia. Also, as part of the Yukos case, three separate claims by former 
Yukos shareholders were filed by Hulley Enterprises Limited (Cyprus), Yukos Universal Limited (Isle of Man), and Veteran Petroleum Limited (Cyprus). As a result, there are only six truly different tax disputes that resulted in a finding of expropriation.

The Señor Tza Yap Shum v. Peru dispute offers a comprehensive illustration of the type of problematic interactions between a taxpayer and an administration that may result in an investment dispute. In 2002, Mr. Tza Yap Shum established a $\$ 400,000$ investment and began operating a fish product export business (TSG). However, in 2004, the Peruvian tax authority, after conducting a routine audit, decided that the amounts and values of the raw materials purchased had not been properly declared and might mean that sales had been under-declared. The authority issued a new tax assessment based on a "presumed basis" of $\$ 4$ million and also took so-called interim measures to enforce the tax assessment that had been imposed to secure money for the Treasury. All banks in Peru were directed to retain any funds related to TSG passing to them and to redirect such funds to the tax authority. Almost immediately, TSG's business became inoperable because the company was unable to pay suppliers or receive payments from its customers.

The arbitral tribunal determined that the interim measures amounted to expropriation. The tribunal concluded that the interim measures significantly interfered with the operation of TSG, were imposed in an arbitrary manner, and did not respect the internal rules and guidelines for its own interim measures and that the local regulators did not make any effort to verify whether these rules were followed. Indeed, the actual tax claim might have been justified, but the temporary enforcement measures taken were so damaging to the operations of the company that the tax claim itself was rendered irrelevant because the company could not survive the measures.

The case illustrates that the recovery measures a state takes to collect tax debts are sensitive from the perspective of the protection foreign investors have under IIAs as well as under general international law. Such measures may easily have an expropriatory effect, even when the amount of tax that is recovered is in itself not confiscatory. A tax debt that is not enormous, but that is disputed and hence not finally determined, may be collected by the state with the use of measures that are so drastic and disproportionate that they result in the discontinuation of the investment. A temporary closure of business facilities, the seizure of business assets or bank accounts, or even the temporary imprisonment of executives of the local company on allegations of tax fraud may all result in the investment losing all value and prospects. In this case, the amount of tax due is secondary to the effect of the recovery measures themselves.

In terms of substance, one can observe that four key substantive provisions have been important to conclude a breach of the relevant IIA has occurred, namely, the expropriation clause, the FET clause, the full protection and security (FPS) standard, and the NT provision. 


\subsection{Tax as the Last Barrier to Investment}

Finally, there is a real risk that tax could become "the last trade and investment barrier," either by design or default. This is because tax systems remain national and are likely to remain so for the foreseeable future (even in regional groupings like the EU), but these national tax systems have to operate in an increasingly global environment where cross-border activities are growing in importance, financial markets are highly integrated, large companies increasingly see themselves as truly global corporations, and technology enables firms and individuals to exploit to the maximum in this increasingly borderless world. Consequently, national tax barriers to investment flows remain (see Christians 2012). Some examples are highlighted below.

\subsubsection{National Tax Barriers to Investment Flows}

First, a major problem is the unrelieved double taxation on cross-border income and capital that occurs if the same income is taxed both in the residence state and the source state. This may influence decisions by multinational enterprises (MNEs) as to where to invest (the OECD's BEPS Project addresses this but will increase this risk, at least in the short term, because it will trigger a number of domestic tax reforms across the world).

Second, there remain inconsistencies in the way in which customs, value-added tax (VAT), and direct tax authorities apply transfer pricing rules to cross-border transactions between related parties within multinational groups, and this may lead to significant compliance costs for companies.

Third, there is a risk of creating a climate of tax uncertainty. The emergence of new players, the rapid development of new technologies, the more aggressive approach to tax planning on the part of some MNEs, and the lack of a global consensus on what should be the international tax rules will lead to more tax uncertainty (it now appears unlikely that BEPS will lead to any fundamental review of the core features of the current international tax framework, with the positions of the OECD countries, Brazil, Russia, China, and South Africa (BRICS); other emerging economies; and developing countries diverging. This lack of agreement will, at least in the short term, lead to a period of uncertainty, a lack of coherence, and disputes between countries).

Fourth, some countries are putting in "exit" taxes under both personal and corporate income taxes, and these taxes may decrease the mobility of capital and labor.

Finally, under the leadership of the WTO and the World Customs Organization (WCO), many tariffs and specific excise barriers to cross-border trade in goods and services have been removed, but friction continues, owing to the inconsistent way in which these rules are sometimes applied. 


\subsubsection{The New Horizon: Promoting Cooperation Between Tax Authorities}

The OECD has concluded that the appropriate response to the pressures of globalization is better cooperation between governments. This is the approach the OECD has followed for many years in the direct tax area and with some success. The OECD Model Tax Convention forms the basis for the 3,600 bilateral tax treaties around the world, which minimize frictions between national tax systems (see also Rosenzweig 2012).

The OECD has many other success stories regarding taxation, e.g.

1. It has been at the forefront of promoting cooperation between tax authorities to counter both double taxation and double non-taxation of cross-border income.

2. Its transfer pricing guidelines are now used as the basis for national legislation both in OECD countries and many non-OECD countries.

3. In close cooperation with the EU, it has also done pioneering work on VAT; this started just over a decade ago, and in the long term, it should lead to more effective cooperation between the 160 countries that currently operate VAT/goods and services tax (GST) systems.

4. The Forum on Tax Administration provides a platform for commissioners from more than 40 countries to come together on a regular basis, and this grouping has now become a powerful voice both in shaping the debate on tax administrations across the world and in helping the commissioners to work together to cope with the challenges of globalization.

5. Removing bank secrecy as a barrier to the effective exchange of information between tax administrations.

6. The Base Erosion and Profit Shifting (BEPS) Project in 2013. The main purpose of the BEPS project is to effectively prevent double non-taxation and no or low taxation cases associated with artificially segregated taxable income from its revenue-generating activities.

Nevertheless, the question remains as to whether these forms of non-binding cooperation will be sufficient to avoid tax being used to protect domestic markets, to discriminate in favor of, or against, non-residents, or to give a competitive advantage to a country's enterprises.

\subsection{Conclusion}

This article evidenced an important reality: there are a growing number of international arbitration cases that involve a tax issue. This is not totally surprising. After all, foreign investment decisions and tax regulations are deeply intertwined. However, each was historically regulated by different authorities and agreements and used to belong to different spheres; however, today the spheres overlap. 
In the coming years, such a trend will continue to increase, and because of the shortcomings of the tax dispute resolution mechanisms, many disputes might end up before investment tribunals.

The early jurisprudence of the International Centre for the Settlement of Investment Disputes (ICSID) has already given a strong indication that tax disputes related to foreign investment are also legal disputes that arise directly out of the investment for which the ICSID tribunal may have jurisdiction. Although none of these early cases are directly related to tax matters, tribunals felt it is important to warn the parties that it may one day be appropriate to link investment protection to tax law. In $A M C O$ v. Indonesia, the tribunal observed that tax matters may well be covered by ICSID's jurisdiction. In Kaiser Bauxite v. Jamaica, the government had agreed to a tax stabilization clause, and the tribunal asserted that a dispute over increased taxes would fall under the scope of Article 25 paragraph 1 of the ICSID Convention, because "the dispute concerning the alleged legal rights and obligations stemming from particular provisions in Kaiser's agreements with the Government is a legal dispute." A similar situation and decision was found in Alcoa Minerals v. Jamaica. In this chapter, more recent cases have been reviewed. For example, in Feldman v. Mexico, the issue was the failure of the tax authorities to refund excise taxes for exported cigarettes, which was held by the international arbitration tribunal to be a violation of the NT provision of the IIA. In Occidental v. Ecuador, a case in which the investor was victorious, the dispute sprang from the refusal of the Ecuadorian tax authority to refund input VAT to a foreign investor.

It is important to note that an arbitration tribunal in an international investment case does not sit as a court of appeal to the local tax court or administrative body that decides tax cases in that state. Whether a certain tax is applicable under the laws of a state is a matter for the courts and administrative bodies of that state, not for the arbitration tribunal. The arbitration tribunal decides whether the state breached any international obligations as set out in the IIA, in general international law or, perhaps, in the contract between the state and the investor. In other words, it is not the role of the arbitration tribunal to interpret and apply the tax laws of a state to an investor. But the way a state applies its tax laws, even if applied correctly under that state's law, may very well constitute a breach of the obligations of that state under international law. As such, the matter can be both a question for a local tax court (to be decided solely on the tax laws of that state) and for an arbitration tribunal (to be decided on international investment law).

The last decade has witnessed a dramatic surge in investment disputes between foreign investors and host country governments. Arbitral panels have been charged with the task of applying the rules of IIAs in specific cases, a task which is not often straightforward given the broad and sometimes ambiguous terms of these arrangements. The new phenomenon of investment arbitration has brought about a number of decisions from different arbitral fora in the tax sector, contributing to the formation of a jurisprudence that is elucidating the meaning of key provisions and contributing to the emergence of global economic regulation of tax matters. Importantly, 15 disputes have resulted in significant compensation being paid by host states for breaching IIA commitments by imposing tax measures. The details of these 
15 disputes show that there a number of provisions which have proven decisive to justify the claims of the taxpayers, namely, protection against expropriation, FET, FPS, non-discrimination, the umbrella clause, and procedure. These six investment provisions indirectly constitute part of the international regime of tax matters, which is increasingly being shaped by investment tribunals' awards and international investment agreements.

Acknowledgment We would especially like to thank Irma Mosquera, Shintaro Hamanaka, Xu Yan, Noam Noked, and Karl P. Sauvant for indispensable feedback and comments.

\section{References}

Christians, A. (2009). Your own personal tax law: Dispute resolution under the OECD Model Tax Convention. Willamette Journal of International Law and Dispute Resolution, 17(2), 172-185. Christians, A. (2012). How nations share. Indiana Law Journal, 87(4), 1407-1453.

OECD Model Tax Convention on Income and on Capital: Condensed Version 2017. Retrieved June 19, 2020, from https://doi.org/10.1787/mtc_cond-2017-en

Ring, D. (2010). Who is making international tax policy: International organizations as power players in a high stakes world. Fordham International Law Journal, 33(3), 649-722.

Rosenzweig, A. H. (2012). Thinking outside the (tax) treaty. Wisconsin Law Review, 2012(3), 717-786.

Stephan, P. B. (2010). Comparative taxation procedure and tax enforcement. Oxford: Oxford University Press.

Swenson, C. D., Beaumont, S. J., Bennett, M., \& Conway, K. (2011). Managing cross-border tax disputes. Taxes: The Tax Magazine, 89(6), 57-68.

UNCTAD. International investment agreements navigator. Retrieved June 19, 2020, from https:// investmentpolicy.unctad.org/international-investment-agreements

Vieira, M. (2014). The regulation of tax matters in bilateral investment treaties: A dispute resolution perspective. Dispute Resolution International, 8(1), 63-84.

\section{Cases}

Alcoa Minerals of Jamaica Inc. v. Jamaica, ICSID Case No. ARB/74/2Antoine Goetz et consorts v. République du Burundi, ICSID Case No. ARB/95/3.

Amco Asia Corporation and others v. Republic of Indonesia, ICSID Case No. ARB/81/1.

Archer Daniels Midland Company and Tate \& Lyle Ingredients Americas, Inc. v. The United Mexican States, ICSID Case No. ARB (AF)/04/5.

Burlington Resources Inc. v. Republic of Ecuador, ICSID Case No. ARB/08/5 (formerly Burlington Resources Inc. and others v. Republic of Ecuador and Empresa Estatal Petróleos del Ecuador (PetroEcuador)).

Corn Products International, Inc. v. United Mexican States, ICSID Case No. ARB (AF)/04/1.

Christopher and Nancy Lacich v. Government of Canada, NAFTA.

Duke Energy Electroquil Partners \& Electroquil S.A. v. Republic of Ecuador, ICSID Case No. ARB/04/19.

EDF (Services) Limited v. Romania, ICSID Case No. ARB/05/13.

El Paso Energy International Company v. The Argentine Republic, ICSID Case No. ARB/03/15. 
EnCana Corporation v. Republic of Ecuador, LCIA Case No. UN3481, UNCITRAL (formerly EnCana Corporation v. Government of the Republic of Ecuador).

Enron Corporation and Ponderosa Assets, L.P. v. Argentine Republic, ICSID Case No. ARB/01/3 (also known as: Enron Creditors Recovery Corp. and Ponderosa Assets, L.P. v. The Argentine Republic).

Gottlieb Investors Group v. Government of Canada, NAFTA.

Grand River Enterprises Six Nations, Ltd., et al. v. United States of America, UNCITRAL.

Hulley Enterprises Limited (Cyprus) v. The Russian Federation, UNCITRAL, PCA Case No. AA 226.

Jan Oostergetel and Theodora Laurentius v. The Slovak Republic, UNCITRAL.

Kaiser Bauxite Company v. Jamaica, ICSID Case No. ARB/74/3.

Limited Liability Company Amto v. Ukraine, SCC Case No. 080/2005.

Link-Trading Joint Stock Company v. Department for Customs Control of the Republic of Moldova, UNCITRAL.

Marvin Roy Feldman Karpa v. United Mexican States, ICSID Case No. ARB(AF)/99/1 (also known as Marvin Feldman v. Mexico).

Mobil Corporation, Venezuela Holdings, B.V., Mobil Cerro Negro Holding, Ltd., Mobil Venezolana de Petróleos Holdings, Inc., Mobil Cerro Negro, Ltd., and Mobil Venezolana de Petróleos, Inc. v. Bolivarian Republic of Venezuela, Decision on Jurisdiction.

Nations Energy Corporation, Electric Machinery Enterprises Inc., and Jamie Jurado v. The Republic of Panama, ICSID Case No. ARB/06/19.

Noble Energy, Inc. and Machalapower Cia. Ltda. v. The Republic of Ecuador and Consejo Nacional de Electricidad, ICSID Case No. ARB/05/12.

Occidental Exploration v. Ecuador LCIA Case No. UN3467.

Pan American Energy LLC and BP Argentina Exploration Company v. The Argentine Republic, ICSID Case No. ARB/03/13.

Phoenix Action, Ltd. v. The Czech Republic, ICSID Case No. ARB/06/5.

Plama Consortium Limited v. Republic of Bulgaria, ICSID Case No. ARB/03/24.

Quasar de Valores SICAV S.A., Orgor de Valores SICAV S.A., GBI 9000 SICAV S.A. and ALOS 34 S.L. v. The Russian Federation SCC Case No. 24/2007.

Sergei Paushok, CJSC Golden East Company and CJSC Vostokneftegaz Company v. The Government of Mongolia, UNCITRAL.

Renta 4 S.V.S.A, Ahorro Corporación Emergentes F.I., Ahorro Corporación Eurofondo F.I., Rovime Inversiones SICAV S.A., Quasar de Valors SICAV S.A., Orgor de Valores SICAV S. A., GBI 9000 SICAV S.A. v. The Russian Federation, SCC No. 24/2007.

RosInvestCo UK Ltd. v. The Russian Federation, SCC Case No. V079/2005.

Señor Tza Yap Shum v. The Republic of Peru, ICSID Case No. ARB/07/6.

TCW Group, Inc and Dominican Energy Holdings, L.P. v. The Dominican Republic, UNCITRAL.

Tokios Tokelés v. Ukraine, ICSID Case No. ARB/02/18.

The Rompetrol Group N.V. v. Romania, ICSID Case No. ARB/06/3.

Veteran Petroleum Limited (Cyprus) v. The Russian Federation, UNCITRAL, PCA Case No. AA 228.

Yukos Universal Limited (Isle of Man) v. The Russian Federation, UNCITRAL, PCA Case No. AA 227. 
Open Access This chapter is licensed under the terms of the Creative Commons Attribution 4.0 International License (http://creativecommons.org/licenses/by/4.0/), which permits use, sharing, adaptation, distribution and reproduction in any medium or format, as long as you give appropriate credit to the original author(s) and the source, provide a link to the Creative Commons license and indicate if changes were made.

The images or other third party material in this chapter are included in the chapter's Creative Commons license, unless indicated otherwise in a credit line to the material. If material is not included in the chapter's Creative Commons license and your intended use is not permitted by statutory regulation or exceeds the permitted use, you will need to obtain permission directly from the copyright holder. 